\title{
Emerging in Canada
}

$\mathrm{T}$ HE THEME OF 'NEW AND EMERGING PATHOGENS' IS CURRENT and persistent in infectious diseases in both the scientific literature and the popular press. This focus developed from the National Institute of Medicine Report Emerging Infections, published in 1992 (1), and the Centers for Disease Control and Prevention response Addressing Emerging Infectious Disease Threats: A Prevention Strategy for the United States, in 1994 (2). This latter report proposed specific strategies to improve identification and response to changes in infectious disease epidemiology and morbidity in the United States. In Canada, the Lac Tremblant Declaration (3) addressed the same issues, with specific recommendations to ensure optimal readiness in our country to respond to new infectious disease threats. This issue of the Journal brings the topic of emerging pathogens to the bedside in two case series describing patients with clinical illness with agents designated as 'emerging pathogens' in these reports. Singh et al (pages 184-190) report four cases of hantavirus infection identified in Edmonton, among the first cases reported in Canada. Megran et al (pages 191-195) highlight the clinical spectrum of Chlamydia pneumoniae infection by describing two patients with uncommon presentations of infection with this organism.

The cases of hantavirus pulmonary syndrome reported by Singh et al had clinical presentations consistent with the reports from the larger outbreak in the United States. There is a characteristic prodrome of fever, cough, dyspnea, gastrointestinal symptoms and headache, then tachycardia, tachypnea, hypotension and, at presentation, the laboratory findings of hemoconcentration and thrombocytopenia. The four reported cases range from relatively mild disease through fulminant respiratory failure and death. In all four cases there was a history of mouse exposure. Specific interrogation with respect to mouse or mouse droppings exposure appears now to be an essential part of the history of any patient in Canada presenting with a syndrome of otherwise unexplained rapid respiratory failure.

The two cases of $C$ pneumoniae infection reported by Megran et al present unusual manifestations of infection with this organism. A young woman is described who presents with severe infection complicated by respiratory failure, illustrating that $C$ pneumoniae infection is part of the differential diagnosis of rapidly progressive community acquired pneumonia. The second case describes a patient with extrapulmonary manifestations of $C$ pneumoniae infection, including reactive arthritis and erythema multiforma. These cases suggest that C pneumoniae, similar to Mycoplasma pneumoniae, another common cause of usually mild community acquired pneumo- nia, has the potential to cause illness presenting with protean manifestations.

Both for epidemiological surveillance and to provide optimal patient care, infectious diseases and other physicians in clinical practice must understand and have efficient access to diagnostic methods for these emerging pathogens. In Canada, the provincial laboratory system and Laboratory Centre for Disease Control (LCDC) generally provide this service. A third paper in this issue from Peeling at the LCDC (pages 198203) provides a useful summary of the complex, sometimes confusing topic of $C$ pneumoniae diagnostic tests, including recommendations for current laboratory diagnosis of $C$ pneumoniae infection.

In addition to these case series of two emerging pathogens, practitioners have been repeatedly reminded in the past year that Canada is part of the global community of infection. The outbreak of pneumonic plague in India and the more recent outbreak of Ebola virus in Zaire are the two most obvious examples. While no cases of these illnesses were identified, or even anticipated, in Canada, public health and federal quarantine responses were necessary and time-consuming. The LCDC Report in this issue (page 208-209) addresses zoonotic infections in Canada in the context of emerging infections. In addition, a case of cutaneous diphtheria in an armed forces person returning from assignment in Somalia was reported in the last issue of this journal (4). Thus, these emerging pathogens are not just the epidemiological problems of public health but cause disease in the patients who will present to Canadian physicians, particularly infectious disease specialists, who will not just diagnose and report infections, but will also provide patient care. An understanding of the clinical features of illness, as exemplified by the case series reported in this issue, is essential.

\section{REFERENCES}

1. Institute of Medicine. Emerging Infections: Microbial Threats to Health in the United States. Washington: National Academy Press, 1992.

2. Centers for Disease Control and Prevention. Addressing Emerging Infectious Disease Threats: A Prevention Strategy for the United States. Washington: US Department of Health and Human Services, 1994.

3. Health Canada. Proceedings and Recommendations of the Expert Working Group on Emerging Infectious Disease Issues. Can Commun Dis Rep 1994;20S2.

4. Berih A. Cutaneous Corynebacterium diphtheriae: A traveller's disease? Can J Infect Dis 1995;6:150-2.

\author{
LE Nicolle MD FRCPC \\ Health Sciences Centre \\ Winnipeg, Manitoba
}




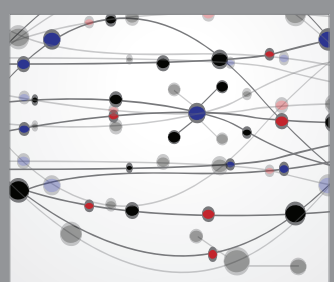

The Scientific World Journal
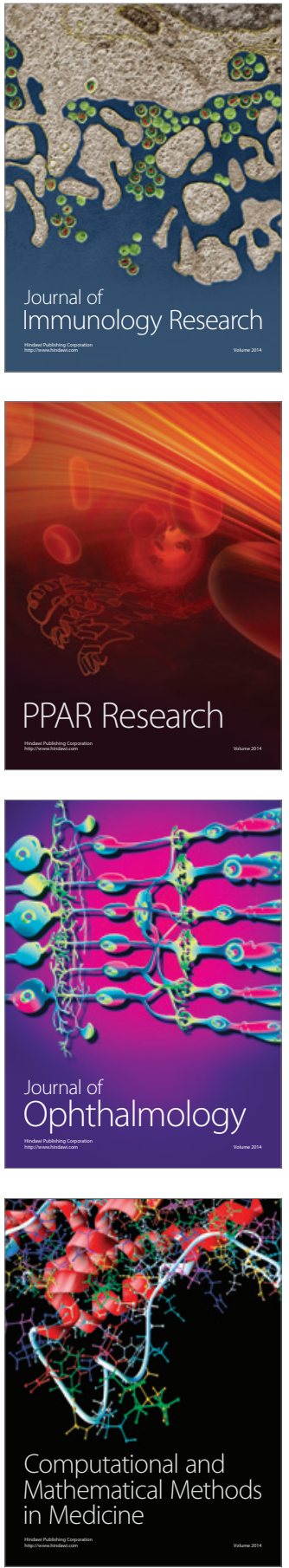

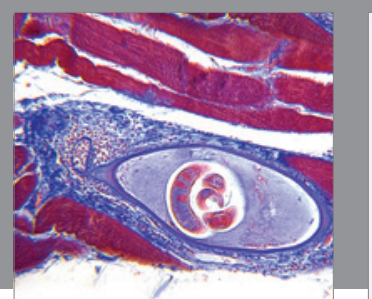

Gastroenterology Research and Practice

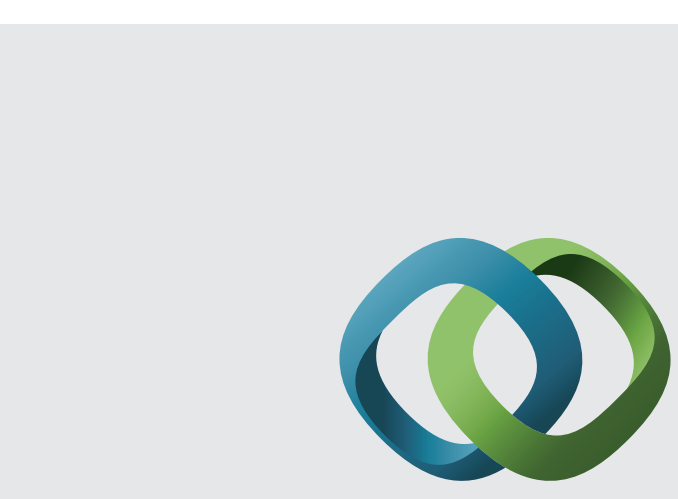

\section{Hindawi}

Submit your manuscripts at

http://www.hindawi.com
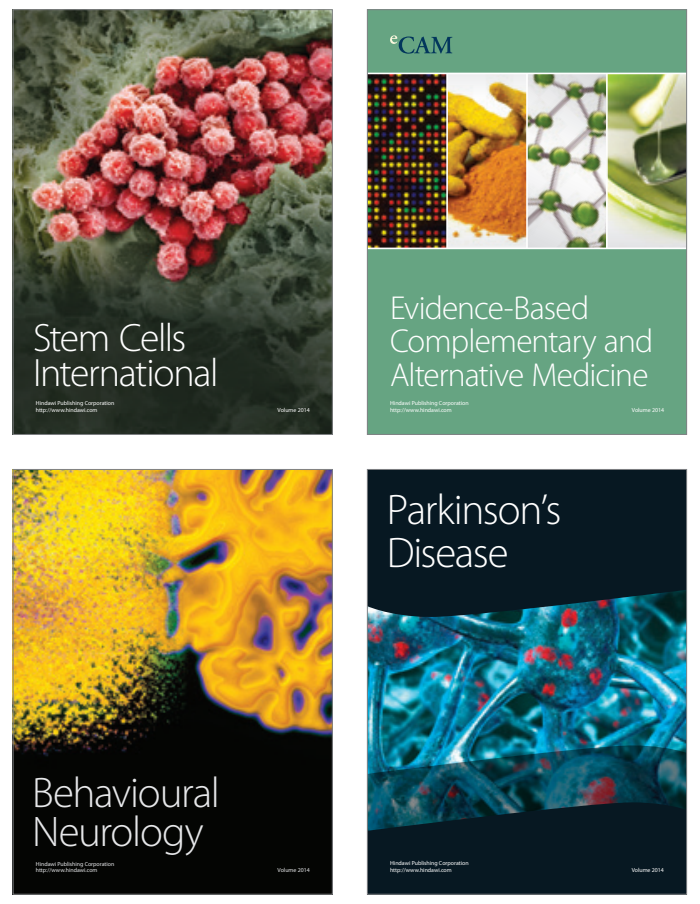
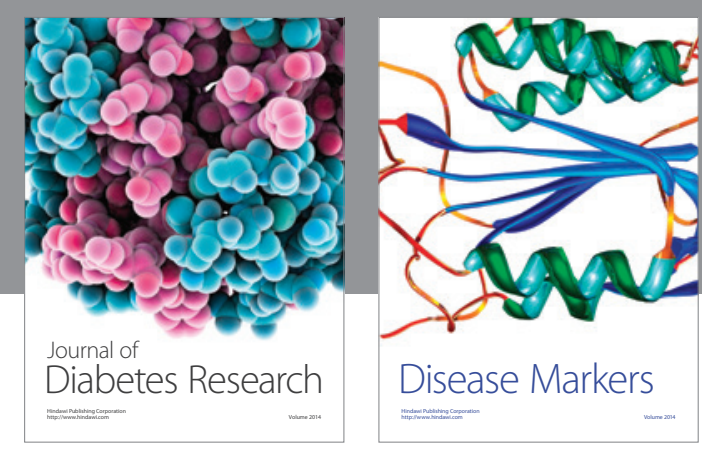

Disease Markers
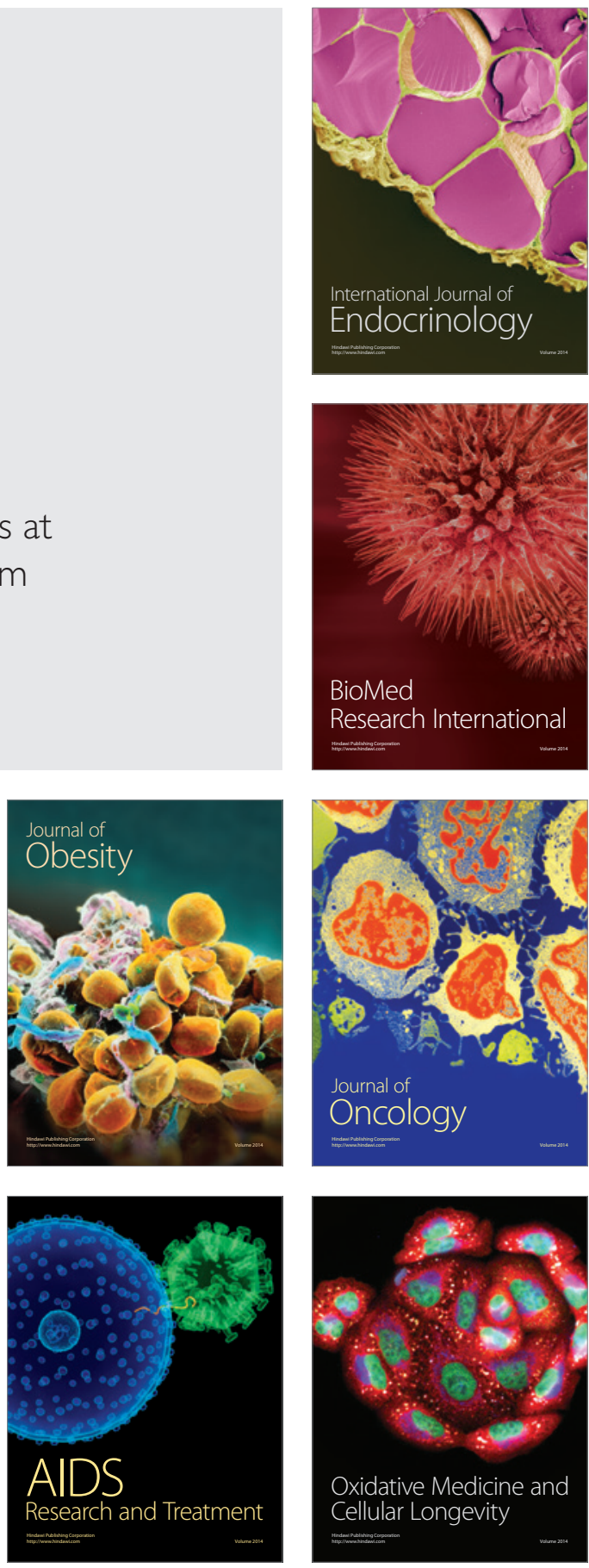